\title{
Subcellular localization of aquaporin 3 in prostate cancer is regulated by RalA
}

\author{
QIWEI CHEN ${ }^{1 *}$, LIANG ZHU $^{2 *}$, HUAFENG ZONG $^{3}$, XISHUANG SONG $^{1}$, LINA WANG $^{1}$, \\ XUEJIAN WANG ${ }^{1}$, DEYONG YANG ${ }^{1}$ and JIANBO WANG ${ }^{1}$ \\ ${ }^{1}$ Department of Urology, First Affiliated Hospital of Dalian Medical University, Dalian, Liaoning 116011; \\ ${ }^{2}$ Department of Physiology, Dalian Medical University; ${ }^{3}$ Department of Pathology, \\ Dalian Municipal Friendship Hospital, Dalian, Liaoning 116000, P.R. China
}

Received August 8, 2017; Accepted February 7, 2018

DOI: $10.3892 /$ or.2018.6308

\begin{abstract}
We previously found that in normal epithelia of the prostate, localization of AQP3 is limited to the cell membranes; however, the expression of AQP3 protein in cancer epithelia is distributed to the plasma. Yet, the detailed mechanism remains unclear. In the present study, PC-3 cell derivatives with stable knockdown of RAS like proto-oncogene A (RalA) and overexpression of E-cadherin were established. We found that overexpression of E-cadherin and knockdown of RaLA resulted in an increase in AQP3 in prostate cancer cell plasma membranes. In order to investigate the functions caused by of the AQP3 redistribution in prostate cancer cells, the growth function of AQP3 redistribution was detected with clonogenic, MTT and MTS assays. In regards to the effect on apoptosis, flow cytometric analysis and DNA Ladder TUNEL assay were utilized. The results showed that AQP3 redistribution in PC-3 cells significantly inhibited the proliferation of cells and enhanced cell apoptosis compared with these parameters in the control. Wound healing assay and Matrigel assays determined that knockout of RalA inhibited the motility and invasion capability of PC-3 cells. To investigate the molecular mechanism involved in AQP3 redistribution in PC-3 cells, the level of cAMP in PC-3 cells was examined, and the results showed that $\mathrm{AQP} 3$ distribution was regulated through cAMP/PKA/RalA signal pathways. In conclusion, these studies suggest a novel function of AQP3, and provide a creative view for RalA-directed therapies.
\end{abstract}

Correspondence to: Professor Deyong Yang or Professor Jianbo Wang, Department of Urology, First Affiliated Hospital of Dalian Medical University, 222 Zhongshan Road, Dalian, Liaoning 116011, P.R. China

E-mail: drcqw21@gmail.com

E-mail:wangjianbo@dlmedu.edu.cn

${ }^{*}$ Contributed equally

Key words: prostate cancer, aquaporin 3, RalA, apoptosis, exocyst, cAMP, distribution

\section{Introduction}

Prostate cancer (PCa) is the most frequent male malignancy and a leading cause of cancer-related death in many Western countries (1). Disease progression in the majority of patients with androgen-dependent prostate cancer can generally be controlled; however, patients with androgen-independent disease and a metastatic phenotype consistently present with a poor prognosis (2).

The molecular mechanisms that underlie the metastatic process and secondary foci specificity are poorly understood. Mainly studies have focused on proteins that function in normal epithelial cells to maintain intercellular adhesion (3). For example, E-cadherin plays an important role in cell-cell adhesion, and a decrease in E-cadherin is associated with cancer progression $(4,5)$. A recent study demonstrated that AQP3, a member of the aquaporins, colocalizes with E-cadherin in the early stages of cell-cell contact formation (6). Aquaporins (AQP) belong to a family of water-transporting proteins, and allow water, glycerol and small solutes through the cell membrane (7). AQP0, 1, 2, 4, 5, 6 and 8 allow the permeation of water. In addition, AQP3, 7, 9 and 10 transport glycerol and small solutes (8).

Our previous study demonstrated that $\mathrm{AQP} 3$ protein is intensely expressed on the cell membranes in normal human prostate cells and tissues, whereas in prostate cancer AQP3 is localized mainly in the cytoplasm (9). Moreover, a recent study showed that after disrupting the exocyst complex, AQP3 distribution to E-cadherin-rich cell-cell contacts was impaired (6). The exocyst, a conserved hetero-octameric protein complex, is localized to lateral membranes and developing apical domains of epithelial cells. In epithelial cells, the exocyst is redistributed from the plasma to areas of cadherin adhesion, and serves to transport post-Golgi transport vesicles to adhesion sites (10). Exocyst distribution and activity are regulated by small GTPases (11). Two of these, Arf6 and Ral, are important promoters of tumor growth and progression (12-14). Among the Ral subfamily, RalA and RalB are highly related GTPases that communicate with parts of the exocyst (Sec5 and Exo84) in vitro (13).

In the present study we explored the function of RAS like proto-oncogene A (RalA) in regulating the localization 
of AQP3 in androgen-independent prostate cancer. We found that depletion of RalA led to the redistribution of AQP3 into the plasma membrane. The tumor-promoting function of RalA in prostate cancer is possibly mediated by the cAMP signaling pathway in prostate cancer.

\section{Materials and methods}

Cell culture and reagents. Human prostate cancer cell line PC-3 was obtained from The American Type Culture Collection (ATCC; Manassas, VA, USA, USA). In this study, we used cells in fewer than 6 months after resuscitation. PC-3 was maintained as monolayer cultures in Gibco F-12 medium (Thermo Fisher Scientific, Inc., Waltham, MA, USA) with $10 \%$ fetal bovine serum (FBS), and incubated at $37^{\circ} \mathrm{C}$ with $5 \% \mathrm{CO}_{2}$ in a humidified atmosphere.

Lentivirus infection. Lentivirus particles carrying shRNA (5'-GATCCGACAGGTTTCTGTAGAAGATTC AAGAGATCTTCTACAGAAACCTGTCTTTTT-3') against RalA or scrambled shRNA (5'-GATCCTTCTCCGAACGTG TCACGTTTCAAGAGAACGTGACACGTTCGGAGAATT TTT-3') (Santa Cruz Biotechnology, Santa Cruz, CA, USA) which has no interference with other cellular mRNA in PC-3 cells were constructed. According to the manufacturer's instructions (Santa Cruz Biotechnology) cells were treated. All functional assays were performed after cell infection.

For DNA transfection, PC-3 cells were grown to $75-80 \%$ confluence and then according to the manufacturer's instructions were transfected with a total of $2 \mathrm{mg}$ of DNA (Life Technologies, Inc., Gaithersburg, MD, USA). E-cadherin expression vector and the neomycin resistance selection marker (Invitrogen; Thermo Fisher Scientific, Inc.) at a ratio of 20:1 were performed. Selective transfectants were tested for transgene expression.

Western blot analysis. For western blot analysis, in brief, protein was extracted using RIPA lysis buffer and protease inhibitor (Thermo Fisher Scientific, Inc.). Thirty micrograms of protein from each sample were separated on $10 \%$ SDS-polyacrylamide gel. GAPDH served as a loading control. Antibodies used included: AQP3 (1:200; rabbit polyclonal antibody; cat. no. ab125219) RalA (1:1,000; rabbit monoclonal antibody; cat. no. ab126627), E-cadherin (1:5,000; rabbit polyclonal antibody; cat. no. ab40772), cAMP (1:30,000; rabbit polyclonal antibody; cat. no. ab76238), T-PKA (1:2,000; rabbit polyclonal antibody; cat. no. ab38949), p-PKA (1:2,500; rabbit monoclonal; cat. no. ab75991) and GAPDH (1:10,000; mouse monoclonal antibody; cat. no. ab8245; all from Abcam, Cambridge, MA, USA).

Cell proliferation assays. For the clonogenic assay, cells were seeded onto $60-\mathrm{mm}$ culture dishes at the density of 1,000 cells/well. After colonies formed ( 10 days), the colonies were fixed, stained (crystal violet; cat no. KGA229; Nanjing KeyGen Biotech, Co., Ltd., Nanjing, China) and were counted [colonies with more than 50 cells were counted under a microscope at x200 magnification (Leica microscope DM4000 B; Leica Microsystems, Wetzlar, Germany)].
For the proliferation assay, cells were seeded onto 96-well plates $(5,000$ per well) in F-12 with 5\% FBS, and cells were treated according to the manufacturer's instructions (Keygentec, Taoshan, China). Absorbance was measured at $550 \mathrm{~nm}$ using Mikrotek Laborsysteme (Mikrotek, Overath, Germany).

In regards to the MTS assay, the cells were analyzed using a CellTiter $96^{\circledR}$ AQueous One Solution Cell Proliferation Assay Kit (Promega, Madison, WI, USA) at $490 \mathrm{~mm}$ absorbance according to the manufacturer's instructions. Doubling times were determined from four replicate samples per point.

Cell cycle distribution assay. Cells were harvested at $48 \mathrm{~h}$ and labeled with propidium iodide (PI) using previously described methods (15). Briefly, cells were resuspended and fixed, and then PI $(0.05 \mathrm{mg} / \mathrm{ml}$; Sigma-Aldrich; Merck KGaA, Darmstadt, Germany) was added to the cells and maintained at room temperature in the dark for $30 \mathrm{~min}$. Cell cycle distribution was examined using a FACScan instrument equipped with FACStation running CellQuest software (Becton Dickinson, San Jose, CA, USA).

Cell apoptosis assay. Apoptosis was assessed by DNA ladder formation, double stained with Annexin V-fluorescein isothiocyanate (FITC)/PI and TUNEL assay. For DNA ladder formation assays, tumor cells were plated in 6-well plates and attached, and then washed twice with PBS, followed by treatment according to the manufacturer's instructions (KGA111; Nanjing KeyGen Biotech Co., Ltd.). A NanoDrop spectrophotometer (Thermo Fisher Scientific) was used to measure DNA concentrations. Total genomic DNA $(5 \mu \mathrm{g})$ was separated on a $1 \%$ agarose gel and photographed with a VersaDoc molecular imager (Bio-Rad Laboratories, Hercules, CA, USA).

For FACS, with or without infection for $48 \mathrm{~h}$, cells were collected and then double-stained with Annexin V-FITC and PI (Becton Dickinson). Cells were examined by a FACScan instrument equipped with FACStation running CellQuest software (Becton Dickinson).

ATUNELassay was conducted to detectapoptosis. The samples were treated with $20 \mu \mathrm{g} / \mathrm{ml}$ non-deoxyribonuclease (DNase) proteinase K (Merck Drugs \& Biotechnology Inc., Darmstadt, Germany) for $30 \mathrm{~min}$ at $37^{\circ} \mathrm{C}$ to remove the nuclease, followed by washing with PBS three times for 4 min each. Terminal deoxynucleotidyl transferase (TdT) (F. Hoffmann-La Roche \& Co., Pleasanton, CA, USA) and biotin-dUTP (F. Hoffmann-La Roche \& Co) were added to the samples, and the mixtures were incubated in the dark at $37^{\circ} \mathrm{C}$ for $60 \mathrm{~min}$, followed by PBS washing. Labeling reaction termination solution was added to the samples, followed by incubation at $37^{\circ} \mathrm{C}$ for $60 \mathrm{~min}$ and washing with PBS. Streptavidin-HRP enzyme (F. Hoffmann-La Roche \& Co.) $(10 \mu \mathrm{l})$ and Biotin-dUTP (490 $\mu \mathrm{l}$ ) were mixed and added onto the sections, and the sections were incubated in the dark for $60 \mathrm{~min}$ at $37^{\circ} \mathrm{C}$. After PBS washing, 3-diaminobenzidine (DAB) solution (F. Hoffmann-La Roche \& Co.) was used to develop the stain. The samples were counterstained with hematoxylin (Solarbio Science \& Technology Co., Ltd., Beijing, China). The samples 


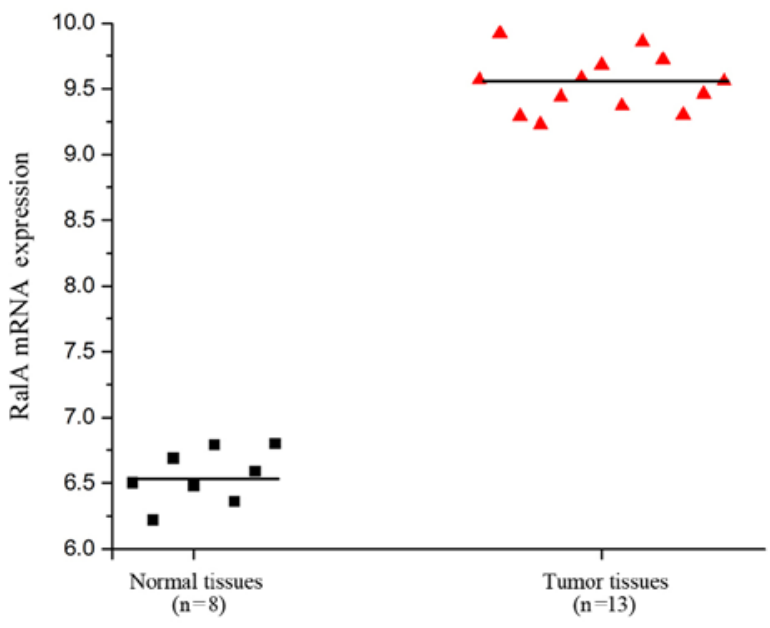

Figure 1. The level of RalA was markedly increased in prostate cancer tissues when compared with the adjacent tissues of patients from the GEO dataset GSE55945 ( $\mathrm{P}<0.05$ as compared with the control). Bars indicate the mean.

were washed with PBS, dehydrated, mounted in neutral resins and photographed (fluorescence microscope Leica TCS SP8; Leica Microsystems).

Wounding healing and invasion assays. Invasion assays were performed in 24-well BD BioCoat ${ }^{\mathrm{TM}}$ Matrigel Invasion Chambers (BD Biosciences, San Jose, CA, USA). After incubation, non-invading cells were removed and the bottom cells were stained with $0.2 \%$ crystal violet, and the stained cells were counted under a microscope (Leica microscope DM4000 B; Leica Microsystems) at x200 magnification, and a bar graph was used to demonstrate the results.

Wound healing assay was performed after transfection as previously described (16). Images of the wound area were captured (Leica microscope DM4000 B; Leica Microsystems) at 0,24 and $36 \mathrm{~h}$.

Immunofluorescence microscopy. Cells were fixed in $4 \%$ paraformaldehyde, and permeabilized using 0.5\% Triton X-100 in PBS and blocked, and then incubated with primary antibody AQP3 (1:250; rabbit polyclonal antibody; cat. no. ab125219; Abcam) and then stained with the secondary antibodies (Rhodamine-labelled secondary antibodies; cat. no. R-6394; Invitrogen; Thermo Fisher Scientific, Inc.). Nuclei were counterstained with DAPI (41,6-diamidino-2-phenylindole). Images were captured on a Leica TCS-SP8 fluorescence microscope (Leica Microsystems).

Statistical analysis and gene set enrichment analysis (GSEA). All data are shown as the mean \pm SD. Statistical analysis was performed with SPSS 17.0 (IBM, Armonk, NY, USA) and OriginPro 8.0 (Originlab, Northampton, MA, USA). Differences in mean values between two groups were analyzed by two-tailed Student's t-test and the mean values of more than two groups were compared with one-way analysis of variance (ANOVA). A P-value $<0.05$ was considered to indicate a statistically significant result. The prostate cancer dataset was downloaded from the NCBI Gene Expression Omnibus database, access ID: GSE55945).
A
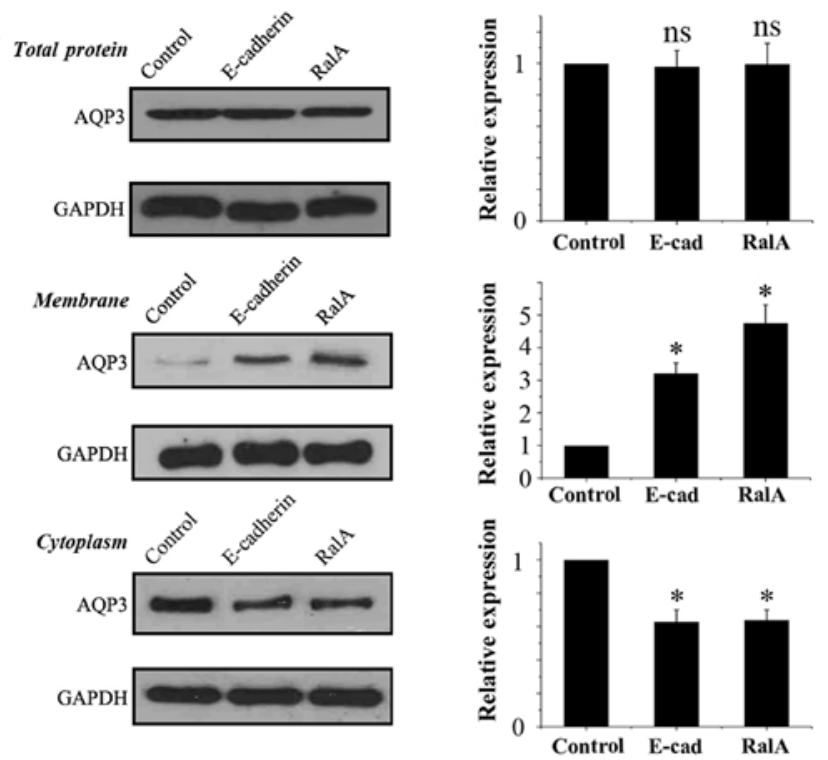

B
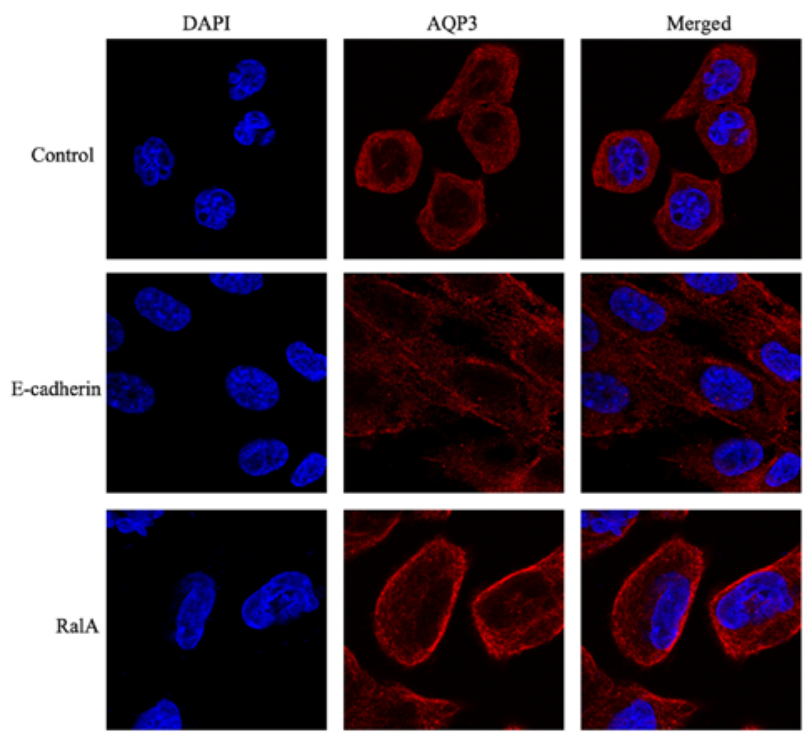

Figure 2. (A) Localization of the expression of AQP3 in PC-3 cells was detected with cell fraction assay. (B) Localization of AQP3 observed by confocal immunofluorescence. E-cad, cells with E-cadherin overexpression; RalA, RalA-knockdown cells. Three independent experiments were performed, and data are shown as mean \pm SD. NS, not significant; ${ }^{*} \mathrm{P}<0.05$ ).

\section{Results}

Downregulation of expression is correlated with the subcellular localization of AQP3 in PC-3 cells. According to the GEO dataset (Access ID: GSE55945), we found an increase in expression levels of RalA in prostate cancer tissues compared with level in the adjacent tissues of patients (Fig. $1, \mathrm{P}<0.01$ ). This result suggests a relationship between RalA mRNA expression and prostate cancer progression.

To further explore this phenomenon, PC-3 cell derivatives with stable knockdown of RalA were established. Moreover, according to Nejsum and Nelson (6), we established PC-3 cell derivatives with stable overexpression of E-cadherin as a positive control.

As shown in Fig. 2A, immunoblot analysis for total AQP3 in the three groups demonstrated that RalA knockdown and E-cadherin overexpression played no significantly role in 

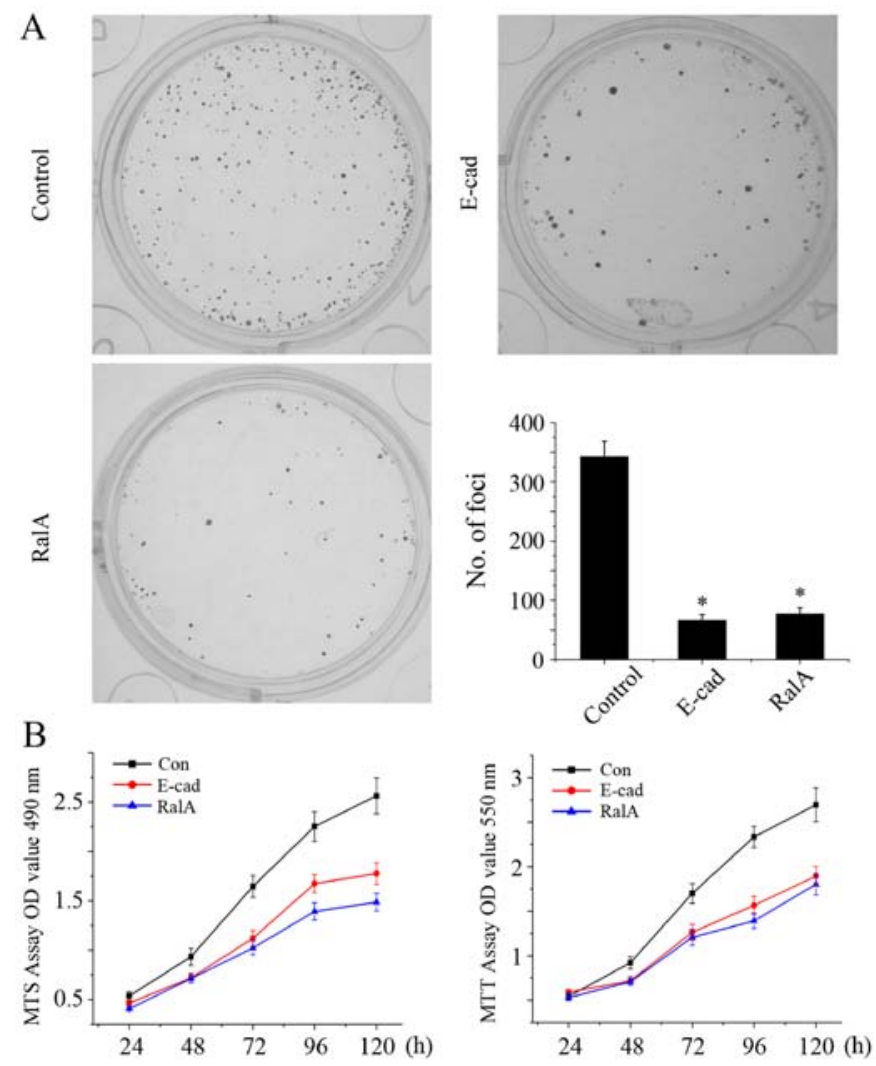

Figure 3. (A) Colony formation assay in monolayer culture of the three groups of PC-3 cells. Cells were stained with crystal violet. (B) In the MTS assay (left) and MTT assay (right), both E-cadherin-overexpressing and RalA-knockdown cells showed decreased cell viability compared with the parental cells. E-cad, cells with E-cadherin overexpression; RalA, RalA-knockdown cells. Data were based on 3 independent experiments. ${ }^{*} \mathrm{P}<0.05$.

regulating the total protein level of AQP3. Subcellular fractionation followed by immunoblot analysis showed the AQP3 band in PC-3 cell plasma and intracellular membranes. RalA knockdown and E-cadherin overexpression resulted in an increase in AQP3 in the PC-3 cell membrane and a simultaneous decrease in AQP3 in the plasma. Moreover, confocal immunofluorescence microscopy in PC-3 cells was in agreement with the quantitative immune blot analysis (Fig. 2B). These data suggest that RalA knockdown regulates the subcellular location of AQP3.

Silencing of RalA inhibits the proliferation of prostate cancer cells. After knockdown of RalA, the number of tumor cells was significantly less and smaller sizes of cell colonies formed were noted when compared with the control group (Fig. 3A, $\mathrm{P}<0.01$ ), Moreover, according to the MTT and MTS assays, in the absence of RalA, the cell growth rate was significantly suppressed when compared with corresponding control (Fig. 3B, left and right graphs, $\mathrm{P}<0.01)$.

Knockdown of RalA enhances the apoptosis of prostate cancer cells. We next aimed to explore how RalA affects cell cycle progression. Flow cytometric analysis revealed that the percentage of PC-3 cells in the G0/G1 phase with viral transfection was markedly increased by $21.2 \%$ (Fig. 4A, $\mathrm{P}<0.05)$, and the percentage of $\mathrm{S}$ phase cells was significantly decreased, compared with the control cells. These results indicated that RalA enhanced G1/S cell cycle transition in prostate cancer cells.

We further determined the apoptotic rate after knockdown of RalA in the PC-3 cells by Annexin V-FITC/PI staining assay. As shown in Fig. 4B, flow cytometric analysis revealed that the silencing of RalA and overexpression of E-cadherin in PC-3 cells markedly enhanced cell death compared with that noted in the control group (RalA, $22.5 \pm 1.31 \%$ vs. E-cadherin, $17.9 \pm 1.26 \%$ vs. control, $3.2 \pm 0.33 \%$; $\mathrm{P}<0.01)$.

In the TUNEL assay, we showed that AQP3 redistribution significantly induced cell apoptosis compared with the control (Fig. 4C; RalA, 78.9 \pm 4.5 cells/field vs. E-cadherin, 59.7 \pm 3.8 cells/field vs. control, 9.2 \pm 1.7 cells/field; $\mathrm{P}<0.01$ ).

Moreover, in the DNA Ladder assay, the treatment groups formed a ladder-like trend which suggested that AQP3 redistribution in PC-3 cells markedly enhanced cell death compared with that noted in the control group (Fig. 4D).

Silencing of RalA suppresses the migration and invasion of prostate cancer cells. We then investigated the function of RalA in the migration and invasion in the treated tumor cells. To this end, we conducted wound-healing and Transwell chamber assays. The results showed that RalA knockdown decreased the motility of tumor cells (Fig. 5A). Furthermore, the RalA knockdown group showed suppressed invasion in the Matrigel-coated Transwells when compared with the control group (Fig. 5B).

Ral GTPases stimulate GTP exchange on two substrates, RalA and RalB (17). Moreover, Shipitsin et al indicated that RalA enhances the delivery of vesicles containing E-cadherin between the trans-Golgi and the basolateral plasma membrane (18). In addition, Nejsum and Nelson concluded that basolateral membrane AQP3 is localized directly to initial areas of E-cadherin-mediated cell-cell adhesion (6). Therefore, we investigated that the presence of RalA components has an important effect on AQP3. To further explore the mechanism by which the signaling pathway is involved in regulating AQP3 via RalA, we focused on the PKA-cAMP signaling pathway which was found to regulate AQP8 subcellular location in rat hepatocytes (19).

Forskolin, a direct activator of adenylyl cyclases, was added to test the signaling pathway, The localization of AQP3 and the expression of pathway-associated protein were evaluated by western blot analysis (Fig. 6). As expected, the presence of forskolin $(10 \mu \mathrm{M})$ resulted in less membranous and more cytoplasmatic distribution of AQP3 in the PC-3 cells, compared with the control group. In the shRNA-RalA group, treatment with forskolin redistributed AQP3. In addition, both p-PKA and RalA levels were increased compared with the control group, which indicated that a cAMP-PKA dependent pathway activated RalA and promoted AQP3 distribution.

\section{Discussion}

To the best of our knowledge, this is the first study on the subcellular location of AQP3 protein in prostate cancer cells. Our data suggest that AQP3 is localized 
A
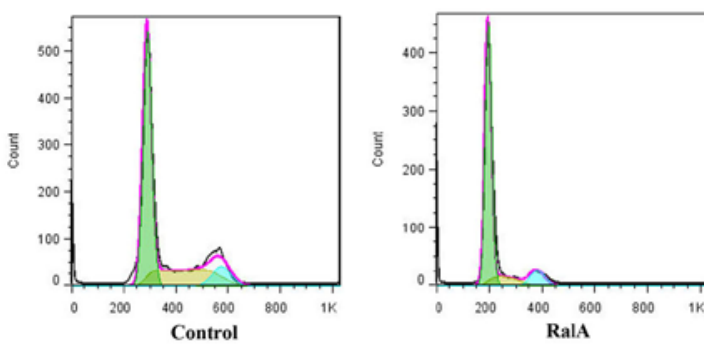

B
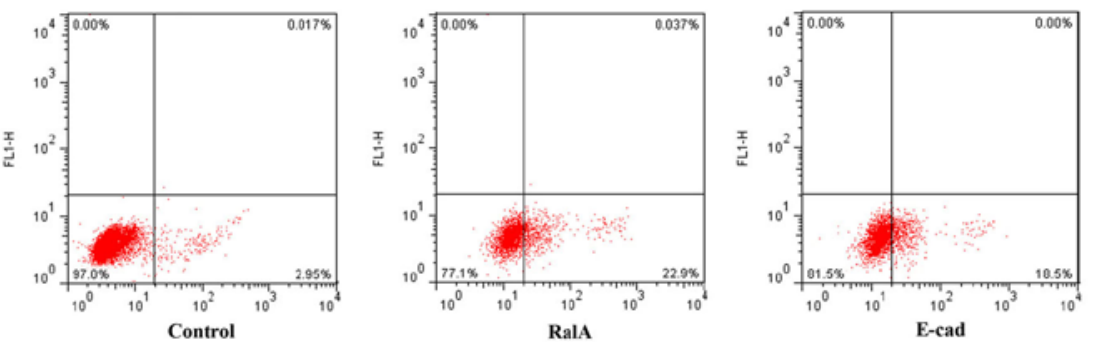
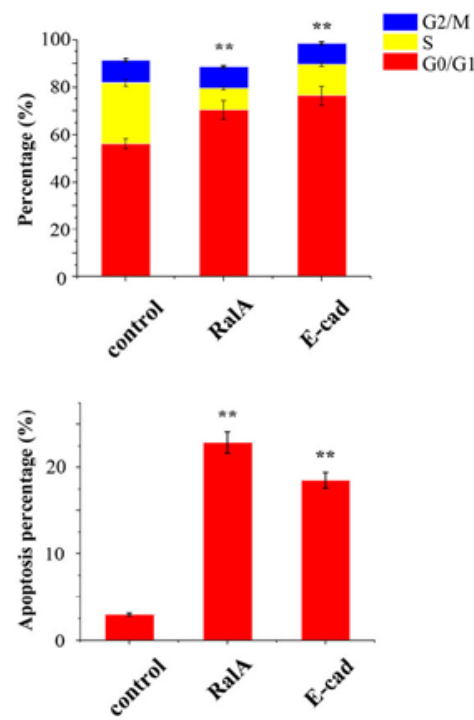

D

C

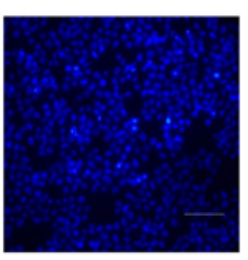

Control

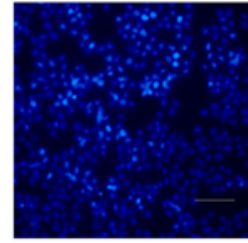

RalA

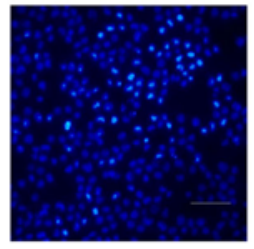

E-cad
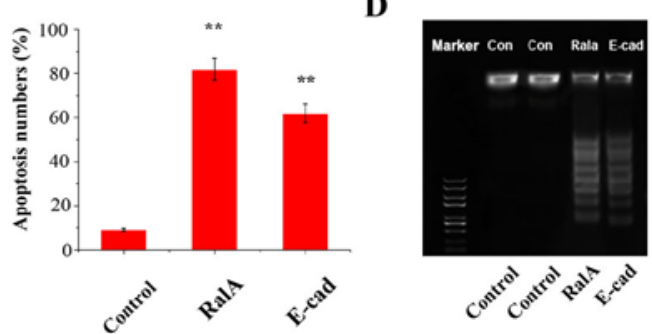

Figure 4. (A) Cell cycle data were analyzed by flow cytometry. Knockdown of RalA and upregulation of E-cadherin suppressed cell proliferation by attenuating G1/S phase transition. Cells were transfected and $48 \mathrm{~h}$ later, the cells were harvested. Data are shown as mean \pm SD. (B) Cells were treated with Annexin V-FITC/PI stain and analyzed by flow cytometry. (C) Apoptotic detection by TUNEL assay. PI (blue) was used to stain the nuclei while FITC (green) was used to label apoptotic cells. Images were captured by a confocal scanning laser microscope. Scale bar, $100 \mu \mathrm{m}$. Data are shown as mean \pm SD. (D) DNA gel electrophoresis. Clear DNA ladder formation was observed in RalA-knockdown and E-cadherin-overexpressing cells. Lane 1, Marker; lanes 2 and 3 , control groups; lane 4, RalA-knockdown cells; and lane 5, E-cadherin-overexpressing cells. Data were from three independent experiments. E-cad, cells with E-cadherin overexpression; RalA, RalA-knockdown cells $\left({ }^{* *} \mathrm{P}<0.01\right)$.

largely in the cytoplasm and can be targeted to the plasma membrane in prostate cancer cells via a cAMP/PKA/RalA pathway.

Aquaporins play an important role in transporting water and small solutes. To date, 13 subtypes (AQP0-AQP12) have been detected in mammals $(7,8)$. Expression of AQPs promotes disease progression and can predict the poor survival of cancer (8). Increased levels of AQP3 have been detected in squamous cell carcinoma of the skin, pulmonary adenocarcinoma, gastric cancer and colorectal adenocarcinoma (20-23). However, few studies have focused on the subcellular location change of AQP3 between normal prostate tissues and prostate cancer.

Nejsum and Nelson demonstrated that AQP3 targets directly to E-cadherin-mediated cell-cell contacts (6). Moreover, active RalA enhances the rate of delivery of E-cadherin (18). Therefore, we focused our efforts on investigating RalA in prostate cancer.

Ras-like (Ral) GTPases include RalA and RalB, which both have differential functions (24). In several types of cancers, increased expression of RalA has been identified (25-27). Consistent with these studies, according to the GEO dataset (Access ID: GSE45016), we found increased expression levels of RalA in prostate cancer tissues. Moreover, when we silenced RalA in PC-3 cells, we found that the intracellular AQP3 was re-localized to the cell membrane, thereby confirming our hypothesis. In addition, we knocked down RalA in PC-3 cells and found significantly reduced colony formation and cell proliferation, as well as suppression of G1/S cell cycle phase cells and enhanced cell apoptosis. This was consistent with a study by Ismail et al who found that cryotherapy also redistributed the localization of AQP3 and enhanced the sensitivity of prostate cancer cells to cryotherapy treatment (28). In addition, RalA knockdown suppressed migration and invasion of tumor cells.

The mechanism underlying the regulation of AQP3 subcellular location in prostate cancer remains unclear. We suggest that localization of AQP3 expression was regulated by the cAMP/PKA/RalA signaling pathway. This signaling pathway has been considered as a regulator of cellular migration, invasion and metastasis $(29,30)$. Our data showed that enhanced adenylyl cyclases activation increased RalA expression in shRNA-RalA cells, indicating an important role for the cAMP-PKA-RalA pathway in regulating AQP3 subcellular location.

In summary, we found that RalA was overexpressed in prostate cancer and plays an important role in regulating localization of AQP3 expression and further enhances the proliferation, apoptosis and metastasis of prostate cancer cells. The localization of AQP3 expression may be regulate 

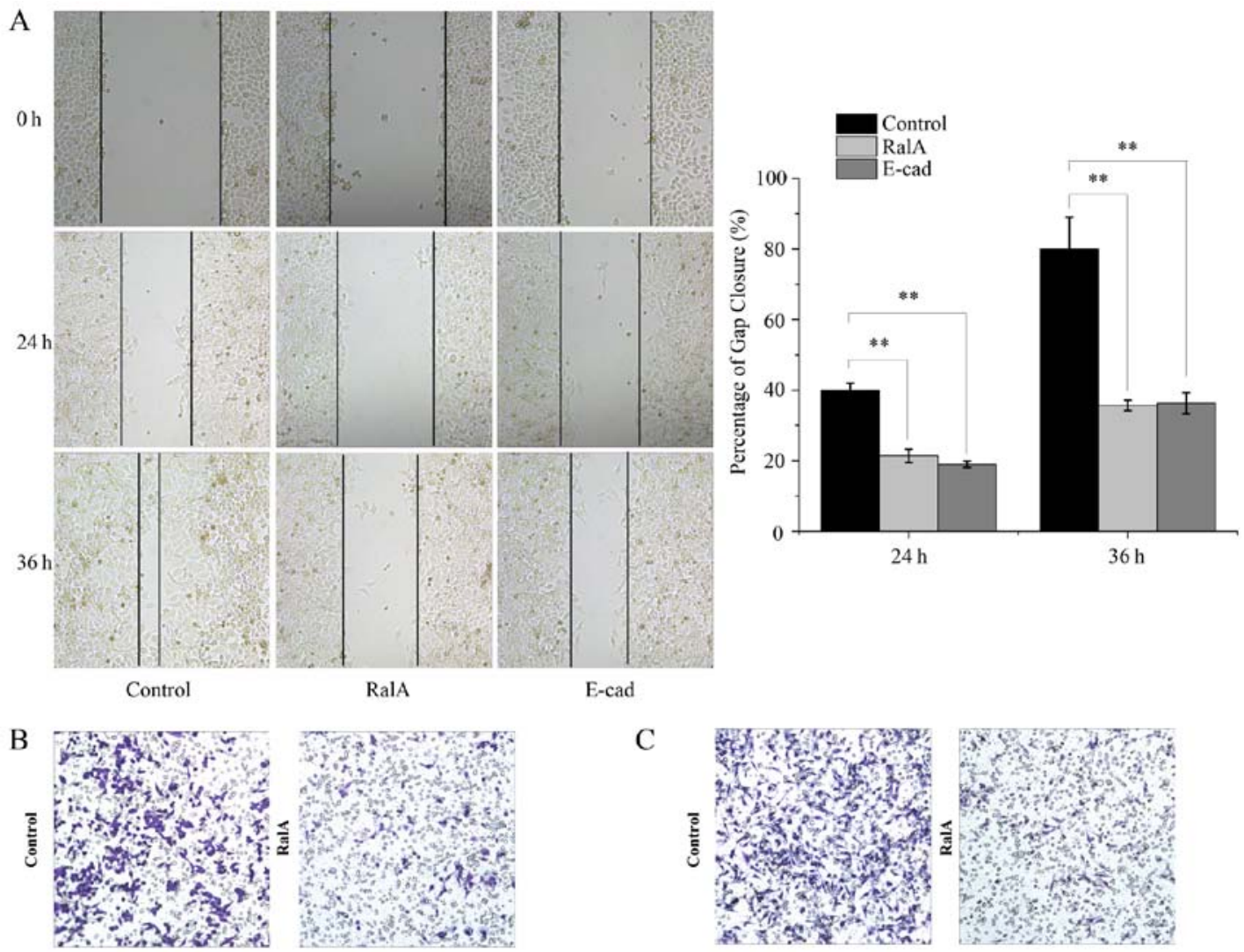

C
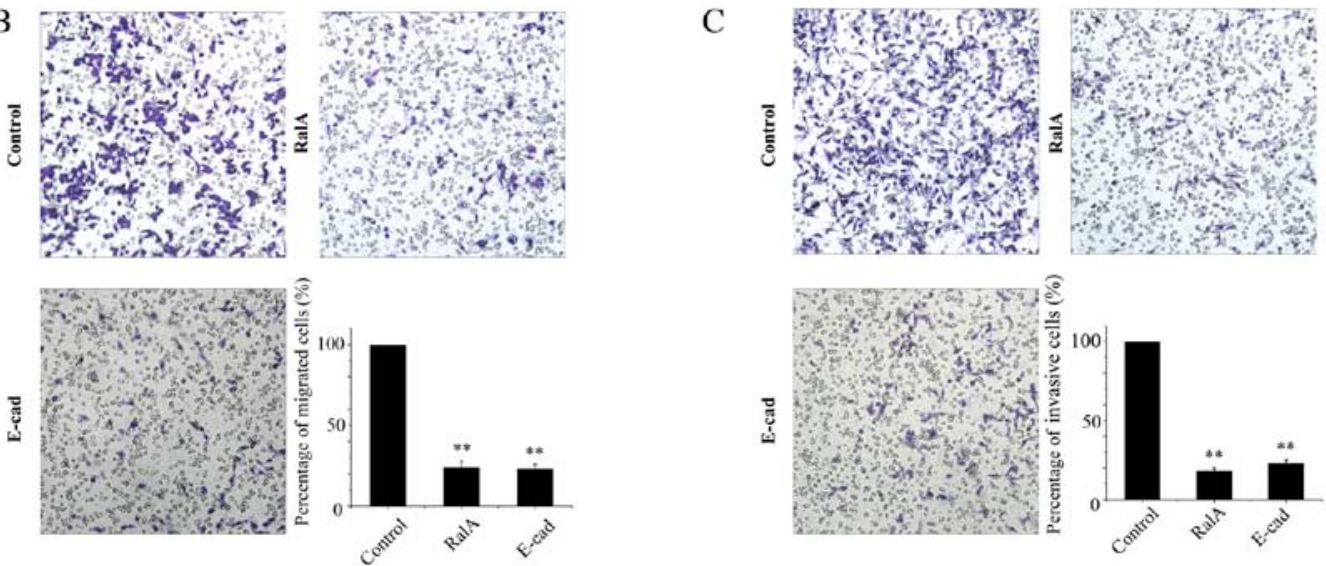

Figure 5. (A) Confluent cell monolayers were wounded with a pipette tip and images were captured at 0,24 and $36 \mathrm{~h}$. Cell migration and invasion were analyzed in Transwell (B) and Matrigel-coated chambers (C). The numbers of migratory and invaded cells were calculated in four selected fields (total space is $2 \mathrm{~mm}^{2}$ ) ( $\mathrm{P}<0.05$ as compared with control); the total number of cells from the control group was set as $100 \%$. E-cad, cells with E-cadherin overexpression; RalA, RalA-knockdown cells. ${ }^{* *} \mathrm{P}<0.01$.
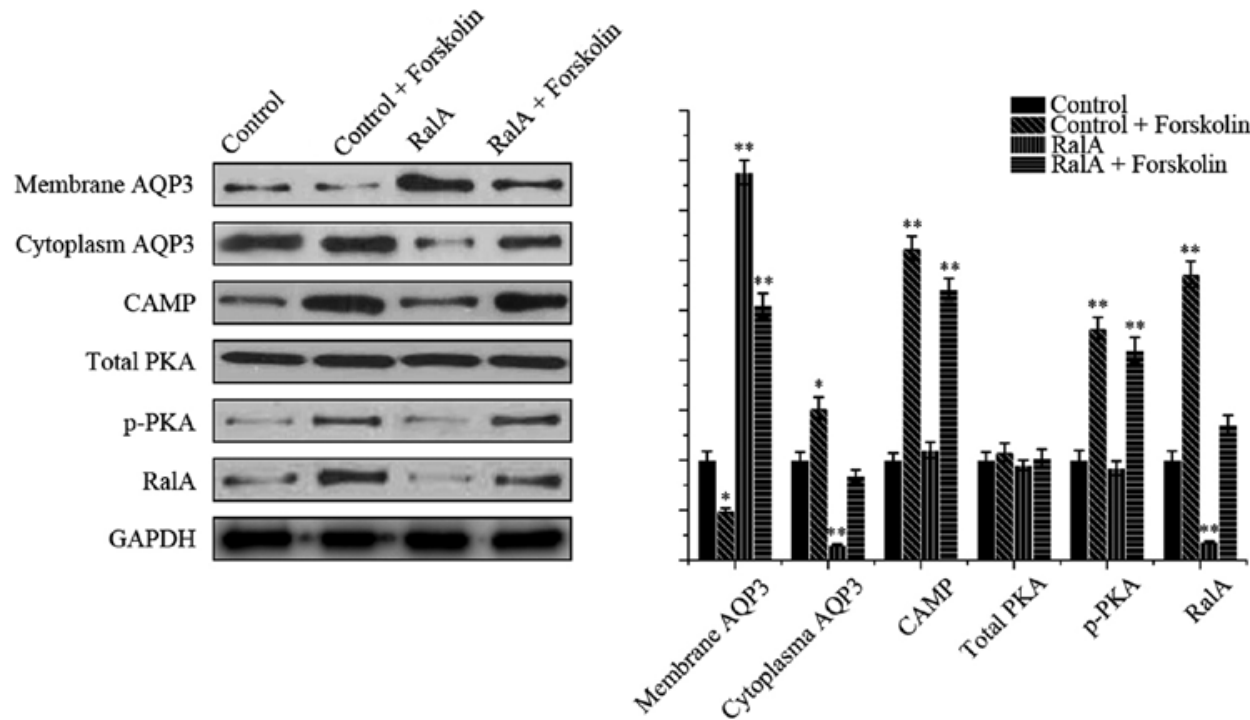

Figure 6. Forskolin stimulates cAMP production and increases RalA protein level in the control group. In the RalA-knockdown cells, Forskolin redistributed AQP3 subcellular location and elevated RalA expression. These results indicate that AQP3 is regulated by the cAMP/PKA/RalA signaling pathway. RalA, RalA-knockdown cells. Data are based on 3 independent experiments, and are shown as mean $\pm \mathrm{SD} .{ }^{*} \mathrm{P}<0.05,{ }^{* *} \mathrm{P}<0.01$. 
by these biological processes through the cAMP/PKA/RalA signaling pathway. These data provide evidence for targeted therapy.

\section{Acknowledgements}

This study was supported by grants from the National Natural Science Foundation of China (nos. 81372761 and 31570953).

\section{Competing interests}

The authors declare that they have no competing interests.

\section{References}

1. Siegel R, Naishadham D and Jemal A: Cancer statistics, 2013. CA Cancer J Clin 63: 11-30, 2013

2. Ruscetti M, Quach B, Dadashian EL, Mulholland DJ and $\mathrm{Wu} \mathrm{H}$ : Tracking and functional characterization of epithelial-mesenchymal transition and mesenchymal tumor cells during prostate cancer metastasis. Cancer Res 75: 2749-2759, 2015.

3. Egan A, Dong Y, Zhang H, Qi Y, Balk SP and Sartor O Castration-resistant prostate cancer: Adaptive responses in the androgen axis. Cancer Treat Rev 40: 426-433, 2014.

4. Wu YC, Tang SJ, Sun GH and Sun KH: CXCR7 mediates TGF $\beta 1$-promoted EMT and tumor-initiating features in lung cancer. Oncogene 35: 2123-2132, 2016.

5. An HT, Yoo $S$ and Ko J: $\alpha$-Actinin-4 induces the epithelial-to-mesenchymal transition and tumorigenesis via regulation of Snail expression and beta-catenin stabilization in cervical cancer. Oncogene 35: 5893-5904, 2016.

6. Nejsum LN and Nelson WJ: A molecular mechanism directly linking E-cadherin adhesion to initiation of epithelial cell surface polarity. J Cell Biol 178: 323-335, 2007.

7. Papadopoulos MC and Saadoun S: Key roles of aquaporins in tumor biology. Biochim Biophys Acta 1848: 2576-2583, 2015.

8. Ribatti D, Ranieri G, Annese T and Nico B: Aquaporins in cancer. Biochim Biophys Acta 1840: 1550-1553, 2014.

9. Wang J, Tanji N, Kikugawa T, Shudou M, Song $X$ and Yokoyama M: Expression of aquaporin 3 in the human prostate. Int J Urol 14: 1088-1092, 2007.

10. Yeaman C, Grindstaff KK and Nelson WJ: Mechanism of recruiting Sec6/8 (exocyst) complex to the apical junctional complex during polarization of epithelial cells. J Cell Sci 117 559-570, 2004

11. Prigent M, Dubois T, Raposo G, Derrien V, Tenza D, Rossé C, Camonis J and Chavrier P: ARF6 controls post-endocytic recycling through its downstream exocyst complex effector. J Cell Biol 163: 1111-1121, 2003.

12. Chabu C, Li DM and Xu T: EGFR/ARF6 regulation of Hh signalling stimulates oncogenic Ras tumour overgrowth. Nat Commun 8: 14688, 2017.

13. Pawar A, Meier JA, Dasgupta A, Diwanji N, Deshpande N, Saxena K, Buwa N, Inchanalkar S, Schwartz MA and Balasubramanian N: Ral-Arf6 crosstalk regulates Ral dependent exocyst trafficking and anchorage independent growth signalling. Cell Signal 28: 1225-1236, 2016.

14. Gentry LR, Nishimura A, Cox AD, Martin TD, Tsygankov D, Nishida M, Elston TC and Der CJ: Divergent roles of CAAX motif-signaled posttranslational modifications in the regulation and subcellular localization of Ral GTPases. J Biol Chem 290: 22851-22861, 2015.
15. Henry MK, Lynch JT, Eapen AK and Quelle FW: DNA damage-induced cell-cycle arrest of hematopoietic cells is overridden by activation of the PI-3 kinase/Akt signaling pathway. Blood 98: 834-841, 2001.

16. Chen Q, Yang D, Zong H, Zhu L, Wang L, Wang X, Zhu X, Song $X$ and Wang J: Growth-induced stress enhances epithelial-mesenchymal transition induced by IL- 6 in clear cell renal cell carcinoma via the Akt/GSK-3 $\beta / \beta$-catenin signaling pathway. Oncogenesis 6: e375, 2017.

17. Thomas JC, Cooper JM, Clayton NS, Wang C, White MA, Abell C, Owen D and Mott HR: Inhibition of Ral GTPases using a stapled peptide approach. J Biol Chem 291: 18310-18325, 2016.

18. Shipitsin M and Feig LA: RalA but not RalB enhances polarized delivery of membrane proteins to the basolateral surface of epithelial cells. Mol Cell Biol 24: 5746-5756, 2004.

19. García F, Kierbel A, Larocca MC, Gradilone SA, Splinter P, LaRusso NF and Marinelli RA: The water channel aquaporin-8 is mainly intracellular in rat hepatocytes, and its plasma membrane insertion is stimulated by cyclic AMP. J Biol Chem 276: 12147-12152, 2001

20. Hua Y, Ma X, Liu X, Yuan X, Qin H and Zhang X: Identification of the potential biomarkers for the metastasis of rectal adenocarcinoma. APMIS 125: 93-100, 2017.

21. Xia H, Ma YF, Yu CH, Li YJ, Tang J, Li JB, Zhao YN and Liu Y: Aquaporin 3 knockdown suppresses tumour growth and angiogenesis in experimental non-small cell lung cancer. Exp Physiol 99: 974-984, 2014.

22. Chen J, Wang T, Zhou YC, Gao F, Zhang ZH, Xu H, Wang SL and Shen LZ: Aquaporin 3 promotes epithelial-mesenchymal transition in gastric cancer. J Exp Clin Cancer Res 33: 38, 2014.

23. Ishimoto S, Wada K, Usami Y, Tanaka N, Aikawa T, Okura M, Nakajima A, Kogo M and Kamisaki Y: Differential expression of aquaporin 5 and aquaporin 3 in squamous cell carcinoma and adenoid cystic carcinoma. Int J Oncol 41: 67-75, 2012.

24. Hobbs GA, Der CJ and Rossman KL: RAS isoforms and mutations in cancer at a glance. J Cell Sci 129: 1287-1292, 2016.

25. Padavano J, Henkhaus RS, Chen H, Skovan BA, Cui H and Ignatenko NA: Mutant K-RAS promotes invasion and metastasis in pancreatic cancer through GTPase signaling pathways. Cancer Growth Metastasis 8 (Suppl 1): S95-S113, 2015.

26. Győrffy B, Stelniec-Klotz I, Sigler C, Kasack K, Redmer T, Qian Y and Schäfer R: Effects of RAL signal transduction in KRAS- and BRAF-mutated cells and prognostic potential of the RAL signature in colorectal cancer. Oncotarget 6: 13334-13346, 2015.

27. Wang K, Terai K, Peng W, Rouyanian A, Liu J, Roby KF, Wise AL, Ezzeldin M, Larson J, Woo RA, et al: The role of RalA in biology and therapy of ovarian cancer. Oncotarget, 2013.

28. Ismail M, Bokaee S, Morgan R, Davies J, Harrington KJ and Pandha H: Inhibition of the aquaporin 3 water channel increases the sensitivity of prostate cancer cells to cryotherapy. Br J Cancer 100: 1889-1895, 2009.

29. Ezzeldin M, Borrego-Diaz E, Taha M, Esfandyari T, Wise AL, Peng W, Rouyanian A, Asvadi Kermani A, Soleimani M, Patrad E, et al: RalA signaling pathway as a therapeutic target in hepatocellular carcinoma (HCC). Mol Oncol 8: 1043-1053, 2014

30. Yu T, Yang G, Hou Y, Tang X, Wu C, Wu XA, Guo L, Zhu Q, Luo H, $\mathrm{Du}$ YE, et al: Cytoplasmic GPER translocation in cancer-associated fibroblasts mediates cAMP/PKA/CREB/glycolytic axis to confer tumor cells with multidrug resistance. Oncogene 36: 2131-2145, 2017. 